\title{
Maternal long chain polyunsaturated fatty acid status is associated with child growth
}

\author{
M. S. Mulhern ${ }^{1}$, A. Yeates ${ }^{1}$, E. M. McSorley ${ }^{1}$, J. M. W. Wallace ${ }^{1}$, G. J. Myers ${ }^{2}$, P. W. Davidson ${ }^{2}$, \\ S. W. Thurston ${ }^{2}$, C. F. Shamlaye ${ }^{3}$, J. Henderson ${ }^{3}$, G. E. Watson ${ }^{2}$, E. van Wijngaarden ${ }^{2}$ and J. J. Strain ${ }^{1}$ \\ ${ }^{1}$ Northern Ireland Centre for Food \& Health (NICHE), University of Ulster, Coleraine, BT52 1SA, ${ }^{2}$ University of Rochester, \\ School of Medicine and Dentistry, United States, ${ }^{3}$ Ministry of Health, Seychelles
}

Consumption of fish during pregnancy is known to be beneficial for child neurodevelopment and cognitive function ${ }^{(1)}$. Fish is the major dietary source of the long chain polyunsaturated fatty acids (LCPUFA), particularly the $n$-3 LCPUFA, docosahexaenoic acid (DHA). DHA is essential for brain growth and function and the human body can synthesise only small amounts. We have recently shown that higher maternal $n$-3 LCPUFA status during pregnancy is associated with improved language scores in children at 5 years of age ${ }^{(2)}$. Other studies have suggested that LCPUFA status has an impact on infant growth ${ }^{(3)}$, a factor which is also known to influence neurodevelopment.

The aim of this study was to investigate associations between maternal LCPUFA status and child growth outcomes in a population of high fish consumers.

This study was conducted as part of the Seychelles Child Development and Nutrition Study Cohort 2. Blood samples were taken from pregnant women at 28 weeks gestation $(n=1474)$ and serum fatty acid methyl esters were quantified using GC-MS, following lipid extraction, as previously described ${ }^{(4)}$. LCPUFA measured included linoleic acid (LA), arachidonic acid (AA), alpha-linolenic acid (ALA), eicosapentaenoic acid (EPA), and DHA. In the offspring, various measures of child growth, including gestational age and birth weight were assessed at birth. At 20 months of age, measures of height, weight and head circumference were obtained for the child. Multiple linear regression analyses, controlling for gestational age, were used to test associations between maternal LCPUFA status and child growth outcomes at birth and at 20 months of age.

The mean (SD) age of pregnant women at enrolment to the study was 26 (6) years. Spearman correlation analyses showed that gestational age of the child was significantly correlated with child birth weight $(r=0.454, P<0.0001)$ and child height $(r=0.066$, $P=0.021)$, weight $(r=0.089, P=0.002)$ and head circumference $(r=0.064, P=0.024)$ at 20 months of age.

Maternal AA concentrations significantly predicted height of the child at 20 months $(\beta=0.115, P<0.0001)$.

\begin{tabular}{|c|c|c|c|c|c|c|c|c|}
\hline & \multicolumn{2}{|c|}{ LA } & \multicolumn{2}{|c|}{ DHA } & \multicolumn{2}{|c|}{ Total $n-3$} & \multicolumn{2}{|c|}{ Total $n-6$} \\
\hline & $\beta$ & $P$ & $\beta$ & $P$ & $\beta$ & $P$ & $\beta$ & $P$ \\
\hline Birth weight & 0.058 & 0.012 & 0.020 & 0.397 & 0.018 & 0.427 & 0.051 & 0.028 \\
\hline Child height at $20 \mathrm{mo}$ & 0.096 & 0.001 & 0.141 & $<0.0001$ & 0.140 & $<0.0001$ & 0.114 & $<0.0001$ \\
\hline Child weight at $20 \mathrm{mo}$ & 0.093 & 0.001 & 0.077 & 0.008 & 0.075 & 0.010 & 0.095 & 0.001 \\
\hline
\end{tabular}

Multiple linear regression analysis of maternal LCPUFA status and child outcomes.

Maternal LA and total $n-6$ concentrations significantly predicted weight of the child at birth. Maternal LA, DHA, total $n-3$ and total $n-6$ concentrations significantly predicted child height and weight at 20 months of age. Maternal LCPUFA status did not significantly predict child head circumference at 20 months of age.

These data concur with recent literature ${ }^{(5)}$ and add to the body of evidence demonstrating associations between maternal LCPUFA status during pregnancy and child growth outcomes. Such associations warrant further investigation.

This work was supported by grants from the US National Institute of Environmental Health Sciences, National Institutes of Health and the Government of Seychelles.

1. Hibbeln JR, Davis JM, Steer C, et al. Lancet 2007;369(9561)578-585.

2. Strain JJ, Davidson PW, Thurston SW, et al. J Nutr 2012;142(11):1943-1949.

3. Hadders-Algra M. Adv Exp Med Biol 2005; 569: 80-94.

4. Bonham MP, Duffy EM, Wallace JMW et al. (2008) Prostag Leukotr Ess 78, 343-350.

5. Moon RJ, Harvey NC, Robinson SM et al. J Clin Endocrinol Metab 2013; 98(1): 299-307. 